\title{
LIENS ENTRE FLEXIBILITÉ DES RÔLES DE SEXE ET EXPLICATIONS DE L'ORIGINE DES DIFFÉRENCES ENTRE SEXES : ÉTUDES CHEZ DES ENFANTS DE 5 À 11 ANS ET DES ADULTES
}

\author{
Manuel Tostain et Joëlle Lebreuilly \\ Groupe d'études de psychologie | Bulletin de psychologie
}

2006/6 - Numéro 486

pages 615 à 627

ISSN 0007-4403

Article disponible en ligne à l'adresse:

http://www.cairn.info/revue-bulletin-de-psychologie-2006-6-page-615.htm

Pour citer cet article :

Tostain Manuel et Lebreuilly Joëlle, « Liens entre flexibilité des rôles de sexe et explications de l'origine des différences entre sexes : études chez des enfants de 5 à 11 ans et des adultes », Bulletin de psychologie, 2006/6 Numéro 486, p. 615-627. DOI : 10.3917/bupsy.486.0615

Distribution électronique Cairn.info pour Groupe d'études de psychologie.

(c) Groupe d'études de psychologie. Tous droits réservés pour tous pays.

La reproduction ou représentation de cet article, notamment par photocopie, n'est autorisée que dans les limites des conditions générales d'utilisation du site ou, le cas échéant, des conditions générales de la licence souscrite par votre établissement. Toute autre reproduction ou représentation, en tout ou partie, sous quelque forme et de quelque manière que ce soit, est interdite sauf accord préalable et écrit de l'éditeur, en dehors des cas prévus par la législation en vigueur en France. II est précisé que son stockage dans une base de données est également interdit. 


\section{Tostain Manuel* LEBREUILLY Joëlle*}

\section{Liens entre flexibilité des rôles de sexe et explications de l'origine des différences entre sexes : études chez des enfants de 5 à 11 ans et des adultes}

\section{INTRODUCTION}

Les rôles de sexe, c'est-à-dire les comportements jugés, d'un point de vue social, comme appropriés pour chaque sexe, contribuent au maintien de différences entre sexes souvent défavorables pour les femmes (Bourdieu, 1998 ; Deaux, Lafrance, 1998). Si l'on souhaite promouvoir une plus grande égalité entre les sexes, il est important de comprendre comment les individus appréhendent les rôles de sexe et leurs origines. Dans ce cadre, l'objectif de cette recherche est d'étudier comment les enfants, par contraste avec les adultes, évaluent les transgressions des rôles de sexe et expliquent les différences entre sexes.

\section{L'adhésion et la tolérance aux transgressions des rôles de sexe selon l'âge}

Les travaux de psychologie du développement (Le Maner-Idrissi, 1997 ; Ruble, Martin, 1998) montrent que très tôt, dès la fin de la deuxième année, les enfants commencent à présenter des comportements sexués, qui se traduisent par des préférences pour les jouets associés à leur groupe de sexe et par des choix affiliatifs privilégiant les interactions avec les pairs de même sexe qu'eux. Cette appropriation des rôles de sexe a lieu à la même période de développement que l'établissement de l'identité sexuée, c'est-à-dire du sentiment d'être une fille ou un garçon (Chiland, 2003).

Cette appropriation des rôles de sexe s'accompagne, selon les individus, de jugements plus ou moins négatifs vis-à-vis des transgressions des rôles de sexe (comme, par exemple, quand une femme exerce un métier habituellement exercé par une majorité d'hommes). Pour ce qui est des enfants, deux tendances développementales différentes sont avancées. La première tendance (Archer, 1984 ; Carter, Patterson, 1982) suggère que plus les enfants grandissent, plus ils acquièrent ce que l'on appelle la flexibilité des rôles de sexe. D'une part, ils deviennent plus tolérants vis-à-vis des transgressions des rôles de sexe en déclarant que celles-ci sont possibles. D'autre part, avec l'âge, les enfants prennent conscience de la relativité culturelle des rôles de sexe, en devenant sensibles au fait que ces rôles peuvent varier selon les sociétés. On explique cette tendance en avançant qu'avec l'âge, les enfants saisissent de mieux en mieux la nature conventionnelle, sociale et arbitraire des rôles de sexe. La seconde tendance (Emmerich, 1982 ; Stoddart, Turiel, 1985) décrit plutôt une évolution en forme de « $U$ », les très jeunes enfants (de 4-5 ans) et les adolescents jugeant plus sévèrement les violations des rôles de sexe que les enfants de 7-8 ans. Ce second pattern développemental s'expliquerait par le fait que les rôles de sexe sont des conventions sociales particulières car elles s'inscrivent dans des enjeux identitaires qui changent avec l'âge (Kohlberg, 1966). Les très jeunes enfants (vers 4-5 ans) auraient une vision rigide et morale des rôles de sexe, c'està-dire déclareraient que ce n'est pas possible, que ce n'est pas bien de les transgresser et ils n'auraient pas conscience de la relativité culturelle des rôles de sexe. Il faut rappeler que la plupart de ces enfants n'ont pas encore atteint ce que l'on appelle la constance de sexe qui correspond à la compréhension que le sexe est une donnée biologique immuable, qu'on ne peut pas changer de sexe (Szkrybalo, Ruble, 1999). Pour les enfants de 4-5 ans, la reconnaissance de l'identité sexuée s'appuie sur le respect des rôles de sexe: par exemple, être un garçon, c'est se comporter comme un garçon. Les très jeunes enfants seraient donc très conformes car pour eux, ce serait le moyen d'être reconnus dans leur identité sexuée (DafflonNovelle, 2004). Par contre, les enfants de 7-8 ans savent que les rôles de sexe ne définissent plus l'identité sexuée. Ils ont acquis la constance de sexe. Dégagés en partie de cette contrainte identitaire, les enfants de cet âge commenceraient à comprendre que les rôles de sexe sont éventuellement modulables selon le contexte. C'est pourquoi, ils feraient preuve de davantage de flexibilité que les enfants plus jeunes (Ruble, Martin, 1998). Un

* UFR de psychologie, Université de Caen, 14032 Caen Cedex, <manuel.tostain@unicaen.fr>. 
nouvel enjeu identitaire expliquerait qu'à partir de l'adolescence, on constate de nouveau une certaine rigidité à propos de l'adhésion aux rôles de sexe. L'adolescence est la période où se développe l'identité sexuelle, c'est-à-dire l'identité dans le rapport amoureux à l'autre, et où commencent les premières expériences sexuelles. Les sujets, à cet âge, considèrent que pour être reconnu, apprécié par les autres, et en particulier par les personnes de l'autre sexe, il est nécessaire de se conformer aux rôles de sexe (Ullian, 1976). À l'adolescence, la rigidité de cette adhésion aux rôles de sexe serait donc une façon d'affirmer une identité sexuelle encore fragile. L'analyse de Levy, Taylor et Gelman (1995) précise ce tableau en distinguant les mesures traditionnelles et évaluatives de la flexibilité des rôles de sexe. Pour ces auteurs, d'un côté les enfants, avec l'âge, acceptent de plus en plus les transgressions des rôles de sexe, en déclarant que ce type de transgressions est possible ou existe dans d'autres pays (mesures traditionnelles). Ici, cette plus grande flexibilité s'expliquerait par l'augmentation des connaissances sociales de l'enfant et par une amélioration de ses capacités cognitives de décentration. Par contre, d'un autre côté, quand il s'agit d'évaluer moralement (« est-ce que c'est bien ? ») ou affectivement ces transgressions (au travers de questions telles que : «aimerais-tu être l'ami de quelqu'un qui fait ce type de transgression?») (mesures évaluatives), il n'y aurait pas nécessairement d'augmentation avec l'âge. Dans ce cas, ce serait parce que cette flexibilité ne relève pas uniquement d'une logique développementale et qu'elle est modulée par des facteurs subjectifs, attitudinaux, personnels et sociaux. Toutefois, cette différence entre aspects traditionnels et évaluatifs de la flexibilité reste à confirmer.

\section{Une tolérance différente selon le sexe}

Si l'évaluation des transgressions des rôles de sexe varie selon l'âge, on peut dire également qu'elle est parfois différente selon le sexe des personnes interrogées. Certains travaux semblent montrer que les filles sont plus tolérantes ou plus favorables aux transgressions des rôles de sexe que les garçons (Archer, 1984; Tap, 1985). Néanmoins, d'autres recherches constatent peu ou pas de différences selon le sexe (Carter, Patterson, 1982 ; Smetana, 1986). Par ailleurs, différentes études mettent en évidence que la transgression des rôles de sexe est souvent mieux acceptée pour les filles que pour les garçons (Le Maner-Idrissi, 1997 ; Levy et coll. 1995 ; Martin, 1990 ; Smetana, 1986). Par exemple, on accepte mieux d'une petite fille qu'elle pratique des activités assignées ou pratiquées plus fréquemment par les garçons, comme jouer à la voiture, que d'un garçon qu'il joue, par exemple, à la poupée. Pour expliquer ce résultat, on avance notamment que les garçons étant traditionnellement plus valorisés que les filles, on est plus attentif à ce qu'ils adoptent des comportements et des attitudes en rapport avec leur groupe de sexe. Ces attentes sociales, plus fortes pour les garçons, passeraient par une attitude plus rigide, par un renforcement plus important des rôles associés à leur sexe. D'où, en définitive, cette moins grande tolérance pour les transgressions des rôles de sexe pour les garçons que pour les filles (Hort, Fagot, Leinbach. 1990). On a là un résultat inverse de ce que l'on constate généralement pour les adultes, où on tolère souvent plus facilement les transgressions des rôles de sexe par les hommes que par les femmes (Eagly, Dickman, 1997). Dans le cas des adultes, on considère généralement que c'est parce que les hommes bénéficient de plus de latitude, de liberté, dans le choix de leurs activités que les femmes (Lorenzi-Cioldi, 2002).

\section{Évolutions enfantines des explications de l'origine des différences entre sexes}

Si les enfants, avec l'âge, font preuve d'une flexibilité plus grande vis-à-vis des rôles de sexe, et comprennent la nature sociale des rôles de sexe, on note également une évolution des explications qu'ils avancent pour rendre compte des différences entre sexes (Ruble, Martin, 1998). D'après Gelman et Taylor (Gelman, 2003; Gelman, Taylor, 2000 ; Taylor, 1996), les enfants présenteraient initialement un biais essentialiste de type biologique, qui consisterait à expliquer les comportements humains en inférant, chez les individus, des propriétés naturelles internes. Ce biais essentialiste, dans le cadre des différences entre sexes, se manifesterait par la tendance des enfants à considérer qu'il est dans l'essence des femmes et des hommes de se comporter de manière spécifique. Les différences constatées entre sexes auraient un fondement naturel, biologique et interne et ne seraient donc pas modifiables par l'environnement. Ce ne serait qu'à la pré-adolescence (vers 12-13 ans) que les enfants prendraient en compte les déterminants sociaux. Ainsi, les explications biologiques s'opposeraient initialement aux explications sociales, les premières diminuant ensuite avec l'âge, tandis que les secondes augmenteraient. Certains travaux vont dans le sens de cet essentialisme biologique initial avancé par Gelman et Taylor (Martin, Parker, 1995 ; Smith, Russell, 1984 ; Ullian, 1976). D'autres auteurs pensent, au contraire, que ce sont les explications psychologiques et sociales qui sont initialement présentes. Par exemple, pour Carey (Astuti, Solomon, Carey, 2004; Carey, 1985, 1995 ; Johnson, Solomon, 1997), qui se situe dans la filiation des travaux de Piaget (1926) et de Kohlberg (1966), l'enfant, dès 4-5 ans, analyse le monde 
qui l'environne et explique les comportements sexués, davantage en termes psychologiques (de désirs, de vouloirs) ou sociaux (ce qui se fait ou ne se fait pas) qu'en termes biologiques (liés à des dimensions physiques, internes). Pour cet auteur, les explications qu'elle qualifie de psychologiques, et qui consistent à considérer que les comportements relèvent de la volonté de chacun, de choix personnels, diminueraient avec l'âge, car ces explications résulteraient initialement d'un certain égocentrisme enfantin qui sous-estime le poids des influences sociales dans la détermination des comportements sexués. Quant aux explications de nature essentialiste, toujours pour cet auteur, elles seraient peu présentes chez les très jeunes enfants car elles supposent de se constituer un savoir biologique qui s'acquière notamment par l'école. Les recherches sur la constance de sexe vont dans le sens de l'interprétation de Carey en montrant que les aspects sociaux peuvent être plus importants que les aspects biologiques chez les jeunes enfants (Bem, 1989 ; Lloyd, Stroyan, 1994 ; Martin, Ruble, Szkrybalo, 2002 ; Szkrybalo, Ruble, 1999). Dans ces travaux, quand on présente deux photographies, l'une d'un garçon nu, l'autre de ce même garçon habillé en jupe (habit culturellement dévolu aux personnes de sexe féminin), on constate que certains enfants (jusqu'à 4-5 ans, parfois 7 ans), s'ils reconnaissent que l'enfant possède un pénis, déclarent néanmoins qu'il s'agit d'une fille. Autrement dit, ces enfants se laissent influencer par ce qui est immédiatement perceptible et associé à la dimension sociale (les vêtements selon les sexes), et ne prennent pas en compte la dimension biologique typique de l'essentialisme évoqué par Gelman et Taylor.

D'autre part, quelques études se sont intéressées aux différences entre filles et garçons pour ce qui concerne l'explication des différences entre sexes. Les résultats ne sont pas homogènes (Ruble, Martin, 1998). Une recherche de Smith et Russell (1984) montre que dès 7 ans, les filles font un peu plus référence que les garçons aux déterminants sociaux des différences entre sexes. On explique cette différence en indiquant que les filles sont, dans une certaine mesure, moins favorisées que les garçons, ce qui les conduirait à être plus sensibles à la relativité, à l'arbitraire des différences entre sexes. Néanmoins, dans les études déjà citées de Carter et Patterson et de Gelman et Taylor, on ne constate pas de différences entre sexes.

\section{Liens entre flexibilité des rôles de sexe et explications des différences entre sexes}

La question qui se pose est de savoir si la façon dont les enfants expliquent l'origine des différences entre sexes s'associe ou non à des attitudes différentes à l'égard des rôles de sexe (Helwig, 2002).
Les études sur cette question sont rares. Chez les adultes, certains auteurs (Bourdieu, 1998 ; LorenziCioldi, 2002) avancent que les explications biologiques, dans la mesure où elles inscrivent les différences entre sexes dans une donnée de nature qu'on ne peut pas, par définition, modifier, orientent vers, et légitiment, une vision relativement figée et traditionnelle des rôles de sexe (voir aussi Martin, Parker, 1995). À l'opposé, les explications sociales, en mettant l'accent sur le caractère construit et arbitraire des différences entre sexes, relativiseraient ces différences et s'associeraient à une perception plus flexible des rôles de sexe. Une recherche expérimentale récente de Neff et Terry-Schmitt (2002), avec des adolescents et des adultes, va dans le sens de ces interprétations. Ces auteurs montrent que les explications biologiques s'associent à des conceptions rigides des rôles de sexe tandis que les explications sociales sont reliées à des attitudes plus flexibles. Néanmoins, ces liens restent à confirmer car, dans cette recherche, ils ne sont pas toujours statistiquement significatifs. Par ailleurs, la question qui reste en suspens est de savoir si l'on constate également ce type de liens chez les enfants.

\section{PRÉSENTATION GÉNÉRALE DE LA RECHERCHE}

L'objectif de cette recherche était d'étudier l'évolution et les liens entre flexibilité des rôles de sexe et types d'explications de l'origine des différences entre sexes. Deux études ont été entreprises. La première étude avait pour but de mettre en évidence des évolutions relativement fines en prenant en compte des enfants âgés respectivement de 5, 7, 9 et 11 ans. La deuxième étude avait pour objectif de comparer les enfants et les adultes. Dans ce cadre, nous avons présenté des transgressions des rôles de sexe et les sujets devaient indiquer leur point de vue. Compte tenu des remarques de Levy et coll. (1995), la flexibilité des rôles de sexe a été appréhendée à la fois par des mesures traditionnelles (possibilité de transgresser la règle et relativité culturelle) et des mesures évaluatives (attitude morale et réaction affective). D'autre part, nous avons donné aux sujets la possibilité d'avoir des réponses intermédiaires (de type «c'est ni bien, ni mal», « un peu »), ce qui est rarement le cas dans les études antérieures qui proposent, la plupart du temps, des choix binaires (de type « oui » «non»). Dans un deuxième temps, nous avons abordé la question de l'explication de l'origine des différences entre sexes en proposant différents types d'explications possibles. À côté des deux types d'explications habituelles : biologiques et sociales, nous avons introduit des explications psychologiques. Nous 
avons décidé cet ajout afin d'être plus en correspondance avec les réponses possibles des enfants. En effet, ainsi qu'on l'a mentionné plus haut, Carey met en avant la présence chez les enfants d'explications psychologiques où domine l'idée que les comportements résultent de choix personnels. Enfin, nous avons étudié les liens entre flexibilité des rôles de sexe et types d'explications des différences entre sexes, ce thème ayant été rarement abordé chez les enfants.

\section{Hypothèses}

Hypothèse 1. L'influence de l'âge sur la flexibilité des rôles de sexe dépend du type de mesures utilisées : les mesures traditionnelles mettent en évidence une augmentation de la flexibilité avec l'âge; par contre, les mesures évaluatives ne montrent pas d'évolution de la flexibilité avec l'âge.

Hypothèse 2. Avec l'âge, en raison de la prise de conscience des déterminants sociaux et de la relativité culturelle des comportements sexués, les explications biologiques des différences entre sexes diminuent, les explications sociales augmentent. Quant aux explications psychologiques renvoyant à l'idée de choix personnel, elles diminueront, compte tenu également de cette prise de conscience de la détermination sociale des comportements sexués.

Hypothèse 3. La flexibilité des rôles de sexe est associée positivement aux explications sociales des différences entre sexes, négativement aux explications biologiques. Par contre, nous n'avons fait pas d'hypothèses sur le lien entre flexibilité et explications psychologiques, en raison de l'absence de recherches sur la question.

\section{ÉTUDE 1}

\section{Méthode}

\section{Étude préalable}

Nous avons effectué une étude préalable auprès de 30 enfants de classes de CP (âge variant entre 5 ans et demi et 7 ans), afin de sélectionner le matériel utilisé pour l'étude 1 . Nous avons soumis aux enfants différentes activités clairement sexuées, choisies à partir, d'une part, des recherches sur les enfants (Liben, Bigler, 2002 ; Miller, Budd, 1999 ; Reid, 1995), d'autre part, des données statistiques sur la répartition des emplois entre femmes et hommes (Insee, Droits des femmes, 2001). Nous avons sélectionné ceux qui étaient jugés par au moins $80 \%$ des enfants comme «beaucoup plus pour les filles» ou «beaucoup plus pour les garçons ».

\section{Population}

Quatre-vingt-dix enfants normalement scolarisés : 10 garçons et 10 filles de grande section maternelle (âge moyen $=5$ ans 6 mois ; étendue $=$ 4 ans 11 mois à 5 ans 10 mois), 10 garçons et 10 filles de CE1 (âge moyen $=7$ ans 5 mois ; étendue $=7$ ans à 8 ans 1 mois), 10 garçons et 10 filles de CM1 (âge moyen $=9$ ans 7 mois ; étendue $=9$ ans 1 mois à 9 ans 11 mois), 16 garçons et 14 filles de $6^{\text {e }}$ (âge moyen $=11$ ans 4 mois ; étendue $=11$ ans à 11 ans 10 mois).

\section{Matériel}

- Partie 1. Flexibilité des rôles de sexe. Quatre activités qualifiées de féminines et quatre activités qualifiées de masculines, et comprenant à chaque fois deux loisirs enfantins et deux métiers adultes (présentées sous forme de dessins pour les enfants les plus jeunes) : activités «féminines »: « jouer à la corde à sauter », « jouer à la poupée », « assis$\operatorname{tant}(\mathrm{e})$ maternel(le) », « coiffeur(se) »; activités «masculines»: «jouer au foot», «jouer à la voiture », « garagiste », « couvreur(se) » (une définition des activités était donnée).

- Partie 2. Explications des différences entre sexes. Quatre affirmations se référant à deux activités qualifiées de féminines et à deux activités qualifiées de masculines : «ce sont surtout les filles qui jouent à la poupée », «ce sont surtout les filles qui jouent à la corde à sauter», «ce sont surtout les garçons qui jouent au foot», «ce sont surtout les garçons qui jouent à la voiture». Pour chaque activité, il y avait trois propositions explicatives : une explication biologique (par exemple, pour les garçons, «c'est parce qu'ils sont nés comme ça. Ils sont faits pour aimer jouer au foot»); une explication sociale ( «c'est parce les gens, les parents, apprennent aux garçons à jouer au foot, et on trouve ça bien que les garçons jouent au foot»); une explication psychologique ( «c'est parce que les garçons, ils ont choisi tout seul de jouer au foot. C'est chacun qui décide d'aimer jouer au foot »).

\section{Procédure}

Partie 1. Pour chaque activité, les sujets répondaient aux questions suivantes: question 1 : connaissance des rôles de sexe, par exemple, «qui joue surtout au foot? les garçons ou les filles?» (réponses possibles : «les garçons », « les filles", «les deux »); question 2: possibilité de transgresser les rôles de sexe, par exemple, «si une femme a envie de faire comme travail garagiste, est-ce qu'elle peut?» (réponses possibles: «non », « un peu », « oui »); question 3 : évaluation «morale» de la transgression des rôles de sexe, par exemple, «est-ce que c'est bien qu'une fille joue au foot? ? (réponses possibles : «ce n'est pas bien », «c'est ni bien ni mal », «c'est bien »; 
question 4 : évaluation affective de la transgression des rôles de sexe, par exemple, «qu'est-ce que ça te fait quand tu vois une fille jouer au foot?» (les réponses possibles étaient présentées sous forme de dessins : «visage pas content », « visage neutre », « visage content »); question 5 : relativité culturelle des rôles de sexe, par exemple, « est-ce que tu crois que dans d'autres pays, les filles, elles jouent au foot? (réponses possibles : «non», « peut-être », « oui »).

- Partie 2. Pour chaque activité, les sujets devaient indiquer leur degré d'accord avec les trois propositions explicatives (biologique, sociale, psychologique). Pour chaque proposition, les réponses possibles étaient : «non, pas d'accord», « un peu d'accord », « oui, d'accord ».

\section{Mode de passation}

Les sujets étaient rencontrés individuellement et la passation était orale. L'expérience se déroulait en deux sessions de 20 minutes environ. Deux semaines s'écoulaient entre chaque session. Lors de chaque session, les sujets passaient la moitié de la partie 1 puis la moitié de la partie 2 . En effet, lors de la première session, une moitié des sujets (groupe A) ne répondait qu'aux questions impliquant des cibles de sexe féminin (dans la partie 1, questions sur les activités «masculines » réalisées par des cibles de sexe féminin; dans la partie 2, questions sur les activités «féminines » réalisées par des cibles de sexe féminin), l'autre moitié des sujets (groupe B) étant questionnée uniquement sur les cibles de sexe masculin. Lors de la seconde session, chaque groupe passait les questions relatives aux cibles non évoquées lors de la première session (cibles de sexe masculin pour le groupe A, cibles de sexe féminin pour le groupe B). Cette procédure, au cours de laquelle les deux sexes cibles n'étaient pas évoqués en même temps, mais lors de deux sessions différentes, avait pour but de limiter la possibilité que les sujets ajustent leurs réponses pour un sexe en fonction de celles fournies pour l'autre sexe. Son objectif était de réduire le nombre de réponses équivalentes pour les deux sexes cibles, et qui seraient données uniquement par désirabilité sociale, par souci de ne pas paraître discriminer un sexe par rapport à l'autre. Par ailleurs, pour chaque partie, les questions (partie 1) et affirmations (partie 2) étaient présentées dans un ordre aléatoire, à l'exception, dans la partie 1, de la question 1 (connaissance des rôles de sexe) qui était toujours posée en premier, et de la question 5 (relativité culturelle), toujours posée en dernier ${ }^{1}$. À la fin de l'étude un entretien était organisé et on

1. La question 1, sur la connaissance des rôles de sexe, était posée en premier pour mettre les sujets dans leur contexte social d'appartenance, les questions 2 à 4 évoquait avec les sujets la question des différences entre sexes.

\section{Codage}

Afin d'avoir des résultats comparables à ceux d'études antérieures (Levy et coll., 1995 ; Smetana, 1986), on a procédé au codage suivant :

- Partie 1. Pour la connaissance des rôles de sexe, une réponse incorrecte, c'est-à-dire n'allant pas dans le sens des différences effectivement constatées entre filles et garçons valait 0 (par exemple, répondre «les filles» pour «qui joue surtout au foot ? »), une réponse « les deux » a été codée 0,5 , une réponse correcte, allant dans le sens de ce qui est effectivement constaté, codée 1 (par exemple, répondre «les filles» pour la corde à sauter). Pour les autres questions, une réponse négative a été codée 0 (par exemple, «non », « ce n'est pas bien »), une réponse intermédiaire (par exemple, « un peu », « ni bien, ni mal ») 0,5, une réponse positive 1 (par exemple, « oui », « visage content »).

- Partie 2. Pour chaque proposition, la réponse «non » a été codée 0 , la réponse « un peu » 0,5 , la réponse «oui »1. Comme dans les études antérieures, nous avons procédé à des analyses de la variance.

\section{Résultats}

Des analyses de variance préalables montrent que les résultats des groupes $\mathrm{A}$ et $\mathrm{B}$ ne sont pas statistiquement différents.

\section{Partie 1. Flexibilité des rôles de sexe}

Les analyses de variance mettent en évidence que, pour chaque question, il n'y a pas de différences entre les réponses aux deux loisirs enfantins « féminins », d'une part, et entre les réponses aux deux loisirs enfantins «masculins», d'autre part. La même chose est constatée pour les métiers. Nous avons donc regroupé, dans chaque catégorie (loisirs enfantins et métiers adultes), les deux activités associées, en termes de stéréotypes, au même sexe. Le score variera entre 0 et 2 (notes possibles : $0 ; 0,5$; $1 ; 1,5 ; 2$ ). En conséquence, les résultats pour chaque question, sont fondés sur une analyse de variance 4 (âge des sujets) $\times 2$ (sexe des sujets) $\times 2$ (caractère sexué des activités : «féminines » versus « masculines ») $\times 2$ (type d'activités : loisirs enfantins versus métiers adultes), les deux derniers facteurs étant des mesures répétées. Suite à cette analyse, pour les différentes questions, il n'y a pas d'effet principal, ni d'effets d'interaction du sexe des sujets.

renvoient à des transgressions des rôles de sexe. La question 5 sur la relativité culturelle, qui évoquait d'autres contextes sociaux, était posée en dernier. 
- Connaissance des rôles de sexe

Le niveau de réponses « correctes » (en correspondance avec la réalité des différences entre sexe) est élevé. On note un effet principal de l'âge des sujets $(\mathrm{F}(1,79)=2,87, \mathrm{p}<.041)$, les réponses «correctes» sont plus nombreuses avec l'augmentation de l'âge (5 ans : 1,$56 ; 7$ ans : 1,$58 ; 9$ ans : 1,$62 ; 11$ ans : 1,74. Voir tableau 1). Les comparaisons post-hoc ${ }^{2}$, indiquent cependant que cette augmentation est non significative. Il y a également un effet principal du type d'activités $(\mathrm{F}(1,79)=32,14, \mathrm{p}<.0001)$, les loisirs $(1,79)$ ayant plus de réponses «correctes » que les métiers $(1,47)$.

- Mesures traditionnelles de la flexibilité des rôles de sexe

\section{- Possibilité de transgresser les rôles de sexe}

On note un effet principal de l'âge des sujets $(\mathrm{F}(1,82)=18,94, \mathrm{p}<.00001)$ qui se traduit par une augmentation significative des réponses «flexibles » (acceptation de la possibilité de la transgression) entre 5 ans $(0,98)$ et 7 ans $(1,35 ;$ post-hoc, $\mathrm{p}<.01)$, puis entre 7 ans et 9 ans $(1,84 ;$ post-hoc, $\mathrm{p}<.0009)$, mais pas entre 9 et 11 ans $(1,91 ;$ post-hoc, $\mathrm{p}<.72$, ns $)$.

\begin{tabular}{|c|c|c|c|c|c|c|c|c|c|}
\hline & & \multicolumn{2}{|c|}{5 ans } & \multicolumn{2}{|c|}{7 ans } & \multicolumn{2}{|c|}{9 ans } & \multicolumn{2}{|c|}{11 ans } \\
\hline & & Lois. & Mét. & Lois. & Mét. & Lois. & Mét. & Lois. & Mét. \\
\hline \multirow{2}{*}{$\begin{array}{l}\text { Connaissance } \\
(0: « \text { incorrecte »; } \\
2: « \text { correcte })\end{array}$} & AF & 1,75 & 1,40 & 1,85 & 1,35 & 1,90 & 1,45 & 1,91 & 1,59 \\
\hline & AM & 1,65 & 1,45 & 1,71 & 1,43 & 1,73 & 1,41 & 1,83 & 1,64 \\
\hline \multirow{2}{*}{$\begin{array}{l}\text { Possibilité } \\
\text { transgression } \\
(0: \text { non ; } 2 \text { : oui })\end{array}$} & AF & 0,89 & 1,12 & 1,29 & 1,42 & 1,96 & 1,78 & 1,87 & 1,93 \\
\hline & AM & 0,82 & 1,10 & 1,19 & 1,51 & 2,00 & 1,61 & 1,88 & 1,94 \\
\hline \multirow{2}{*}{$\begin{array}{l}\text { Évaluation morale } \\
(0: \text { pas bien } \\
2: \text { bien })\end{array}$} & AF & 0,58 & 1,38 & 1,62 & 1,58 & 1,17 & 1,27 & 1,33 & 1,83 \\
\hline & $A M$ & 1,15 & 1,26 & 1,57 & 1,24 & 1,38 & 1,12 & 1,52 & 1,19 \\
\hline \multirow{2}{*}{$\begin{array}{l}\text { Évaluation affective } \\
(0: \text { négative } \\
2: \text { positive })\end{array}$} & $\mathbf{A F}$ & 1,05 & 0,99 & 1,30 & 1,35 & 1,00 & 1,12 & 1,04 & 1,37 \\
\hline & AM & 1,03 & 0,98 & 1,16 & 1,17 & 1,24 & 1,01 & 1,19 & 1,02 \\
\hline \multirow{2}{*}{$\begin{array}{l}\text { Relativité culturelle } \\
(0: \text { non ; } 2 \text { : oui) }\end{array}$} & AF & 0,49 & 1,11 & 1,01 & 1,71 & 1,79 & 1,91 & 1,94 & 1,87 \\
\hline & AM & 0,91 & 0,70 & 1,53 & 1,30 & 1,80 & 1,69 & 1,93 & 1,90 \\
\hline
\end{tabular}

Tableau 1. Flexibilité des rôles de sexe selon l'âge. Notes moyennes par question selon le caractère sexué des activités (AF : activités « féminines »; AM : activités «masculines ») et le type d'activités (Lois. : loisir enfantin ; Mét. : métier adulte) (notes comprises entre 0 et 2).

\section{- Relativité culturelle des rôles de sexe}

On remarque un effet principal de l'âge des sujets $(\mathrm{F}(1,82)=20,05, \mathrm{p}<.00001):$ la reconnaissance de la relativité culturelle des rôles de sexe augmente significativement jusqu'à 9 ans, mais pas après (post-hoc 9-11 ans, $\mathrm{p}<.47$, ns). Il y a un effet d'interaction simple caractère sexué des activités $\mathrm{x}$ type d'activités $(\mathrm{F}(1,82)=15,81, \mathrm{p}<.0001)$ : les enfants déclarent plus souvent la relativité culturelle des loisirs «masculins» (par exemple, réponse indiquant que les filles peuvent jouer dans d'autres pays au foot) $(1,53)$ que des loisirs « féminins » (par exemple, garçons pouvant jouer à la poupée) $(1,30 ;$ post-hoc, $\mathrm{p}<.01)$. À l'inverse, les enfants déclarent plus souvent la relativité

2. Pour les comparaisons post-hoc, nous avons utilisé le test de Newman-Keuls. culturelle des métiers «féminins » (par exemple, hommes pouvant être coiffeurs) que des métiers «masculins» (par exemple, femmes pouvant être « couvreuses ») (respectivement 1,64 et 1,40; posthoc, $\mathrm{p}<.01)$.

- Mesures évaluatives de la flexibilité des rôles de sexe

- Évaluation morale de la transgression des rôles de sexe

Il y a un effet principal de l'âge des sujets $(\mathrm{F}(1,80)=4,38, \mathrm{p}<.006)$, qui se manifeste par une augmentation des réponses positives (réponses « c'est bien ») quand on passe des enfants de 5 ans $(1,09)$ aux enfants de 7 ans $(1,50$; post-hoc, $\mathrm{p}<$.014). On note trois effets d'interaction simple : $1^{\circ}$ un effet caractère sexué des activités $\times$ type d'activités $(\mathrm{F}(1,80)=27,90, \mathrm{p}<.0001) ; 2^{\circ}$ un effet 
âge des sujets $\times$ caractère sexué des activités $(\mathrm{F}(1,80)=3,89, \mathrm{p}<.01) ; 3^{\circ}$ un effet âge des sujets $\times$ type d'activités $(\mathrm{F}(1,80)=4,57, \mathrm{p}<.005)$. Si l'on analyse ces différents effets d'interaction, on relève que les notes sont plus positives, pour les métiers, dans le cas d'une transgression des rôles de sexes réalisée par un homme $(1,52)$ que par une femme $(1,20 ;$ post-hoc, $\mathrm{p}<.001)$. À l'inverse, dans le cas d'une transgression relative à un loisir, les enfants réagissent plus positivement s'il s'agit d'une fille $(1,40)$ que d'un garçon $(1,17 ;$ post-hoc, $\mathrm{p}<.006)$. On remarque qu'à 5 ans, contrairement aux enfants plus âgés qui ne font pas de différences (post-hoc non significatifs), les enfants font des évaluations plus positives, d'une part pour les transgressions réalisées par des cibles de sexe féminin $(1,20)$ que pour les transgressions réalisées par des cibles de sexe masculin $(0,99$; post-hoc, $\mathrm{p}<.02)$, d'autre part, pour les transgressions relatives aux métiers $(1,32)$ que pour les transgressions relatives aux loisirs $(0,87 ;$ post-hoc, $\mathrm{p}<.001)$.

\section{- Évaluation affective de la transgression des rôles de sexe}

On ne constate pas d'effet principal de l'âge des sujets $(\mathrm{F}(1,78)=1,36, \mathrm{p}<.26$, ns). Il y a un effet d'interaction simple caractère sexué des activités $\mathrm{x}$ type d'activités $(\mathrm{F}(1,78)=4,48, \mathrm{p}<.03)$ : les enfants expriment des sentiments plus positifs, dans le cas des métiers, pour les transgressions réalisées par des hommes $(1,24)$ que par des femmes $(1,04$; post-hoc, $\mathrm{p}<.02)$.

\section{Partie 2. Explications des différences entre sexes}

- Des analyses de variance montrent qu'il n'y a pas de différences significatives entre les explications aux deux activités «masculines », ni entre les explications aux deux activités «féminines ». Nous les avons donc regroupées. Les scores varieront entre 0 (pas d'accord) et 2 (d'accord). En conséquence, les résultats sont fondés sur une analyse de la variance 4 (âge des sujets) $\times 2$ (sexe des sujets) $\times 2$ (caractère sexué des activités) $\times 3$ (type d'explications), les deux derniers facteurs étant des mesures répétées.

On ne relève pas d'effets principaux ou d'interaction du sexe des sujets et du caractère sexué des activités. On constate un effet principal du type d'explications $(\mathrm{F}(2,156)=135,94, \mathrm{p}<.00001):$ les sujets sont davantage d'accord avec les explications psychologiques $(1,82)$ qu'avec les explications biologiques $(0,76)$ et les explications sociales $(0,46)$ (les comparaisons post-hoc sont toutes significatives entre ces trois types d'explications) (tableau 2). Plus précisément, on relève un effet d'interaction âge des sujets $\times$ types d'explications $(F(6,156)=3,47, p<.003)$ : les enfants de 11 ans sont plus d'accords avec les explications sociales que les autres enfants, tandis que les enfants de 9 ans ont des scores plus élevés aux explications psychologiques que les enfants de 5 ans (post-hoc, $\mathrm{p}<.024)$.

\begin{tabular}{lccc}
\hline & $\begin{array}{c}\text { Explications } \\
\text { biologiques }\end{array}$ & $\begin{array}{c}\text { Explications } \\
\text { sociales }\end{array}$ & $\begin{array}{c}\text { Explications } \\
\text { psychologiques }\end{array}$ \\
\hline 5 ans & 0,97 & 0,31 & 1,62 \\
\hline 7 ans & 0,72 & 0,21 & 1,81 \\
\hline 9 ans & 0,65 & 0,47 & 1,97 \\
\hline 11 ans & 0,73 & 0,90 & 1,90 \\
\hline
\end{tabular}

Tableau 2. Notes moyennes aux trois types d'explications selon l'âge. Notes comprises entre 0 (pas du tout d'accord) et 2 (tout à fait d'accord).

Des analyses de corrélations ( $\mathrm{R}$ de BravaisPearson) montrent un lien entre explications biologiques et explications sociales, qui est positif chez les enfants de 5 et 7 ans (respectivement Rbp 5 ans $=+0,53, \mathrm{p}<.016 ;$ Rbp 7 ans $=+0,48$, $\mathrm{p}<.032$ ), puis qui devient négatif chez les enfants plus âgés (respectivement Rbp 9 ans $=-0,64$, $\mathrm{p}<.007 ; \quad$ Rbp 11 ans $=-0,43, \mathrm{p}<.03)$. En revanche, à tous les âges, les explications psychologiques ne sont pas corrélées aux deux autres explications (Rbp non significatifs).

Liens entre flexibilité des rôles de sexe et explications des différences entre sexes

Nous avons procédé à des calculs de corrélation entre les différentes mesures (traditionnelles et évaluatives) de la flexibilité de la partie 1, et les réponses pour les trois types d'explications de la partie 2. Les corrélations de chaque mesure de la partie $1 \mathrm{avec}$ les réponses pour la partie 2 n'étant pas significativement différentes (test bilatéral sur les différences de corrélation), nous avons regroupé les différentes mesures de la flexibilité en un indice dit de flexibilité totale.

Les résultats montrent qu'il n'y a pas de corrélations significatives entre l'indice de flexibilité totale et le type d'explication. Une analyse de corrélation, âge par âge, montre cependant, à 11 ans uniquement, une corrélation positive entre flexibilité et explications sociales $(\mathrm{Rbp}=+0,46, \mathrm{p}<.02)$.

\section{Discussion}

Si nous revenons sur ces principaux résultats, pour ce qui concerne la flexibilité, nous constatons, en accord avec l'hypothèse 1, qu'il y a un effet systématique de l'âge des sujets pour les mesures traditionnelles. Par contre, pour les mesures 
évaluatives, l'absence attendue d'effet de l'âge est confirmée pour l'évaluation affective mais pas pour l'évaluation morale. D'un autre côté, on relève que les explications psychologiques sont importantes à tout âge et que les explications sociales sont plus fréquentes chez les enfants les plus âgés de l'étude, ce dernier point allant dans le sens de notre hypothèse 2. En revanche, notre hypothèse 3 n'est pas validée car on ne constate pratiquement aucun lien entre flexibilité des rôles de sexe et type d'explications des différences entre sexes (exception faite du lien à 11 ans, qui est cohérent avec notre hypothèse, entre flexibilité et explications sociales).

Cette unique corrélation à 11 ans peut amener à penser, soit que l'hypothèse 3 d'un lien entre flexibilité des rôles de sexe et explications des différences entre sexes n'est peut-être pas valide, soit que le type de mesure utilisé, et qui était assez simple en raison d'enfants très jeunes, n'était pas suffisamment fin pour mettre en évidence ces liens. On peut aussi avancer, notre hypothèse 3 étant fondée sur des travaux avec des adolescents et des adultes, que flexibilité des rôles de sexe et explications de l'origine des différences entre sexes ne sont pas encore coordonnées chez les enfants. Pour vérifier ces différentes possibilités, nous avons mis en œuvre une seconde étude comparant les réponses d'enfants et d'adultes et employant une mesure plus fine sous forme de positionnement le long d'un continuum. Cette nouvelle mesure étant plus délicate à comprendre par des enfants, nous n'avons pas fait appel à des sujets aussi jeunes que dans la première étude, mais des enfants âgés de 7-8 ans.

\section{ÉTUDE 2}

\section{Méthode}

\section{Population}

Soixante adultes (30 étudiantes et 30 étudiants de première année de sciences économiques; âge moyen $=21$ ans 2 mois) et 60 enfants correspondant à la classe d'âge centrale de l'étude précédente (30 filles et 30 garçons âgés de 7-8 ans; âge moyen $=7$ ans 8 mois).

\section{Matériel $^{3}$}

- Partie 1. Flexibilité des rôles de sexe. Trois activités qualifiées de féminines (assistant(e)-maternel(le), sage-femme, secrétaire) et trois activités qualifiées de masculines (routier, capitaine dans l'armée, président-directeur général (une définition des activités était donnée).

3. Le matériel a été choisi, suite à un examen préalable auprès de deux classes de CM1, avec une procédure identique à celle de l'examen préalable de l'étude 1 .
- Partie 2. Explications des différences entre sexes. Six affirmations se référant à trois activités qualifiées de féminines («ce sont surtout les filles qui jouent à la dînette », «ce sont surtout les filles qui font de la danse», «ce sont surtout les filles qui veulent travailler avec des enfants ») et à trois activités qualifiées de masculines (« ce sont surtout les garçons qui jouent à la voiture », «ce sont les garçons qui font du bricolage », «ce sont surtout les garçons qui veulent être pompier»). Pour chaque affirmation, il y avait trois propositions explicatives identiques à celles de l'étude 1 (biologique, sociale, psychologique).

\section{Procédure}

Pour chaque activité, les sujets se positionnaient sur des échelles d'opinion (constituées par des lignes de 9 centimètres, mode de mesure auquel les enfants ont été entraînés. Voir détails plus bas). Afin d'obtenir l'opinion du sujet, on mesurait ensuite, en centimètres, la distance comprise entre l'extrémité gauche de la ligne et la position du sujet sur la ligne.

- Partie 1. Flexibilité des rôles de sexe. Pour chaque activité, les enfants répondaient aux questions suivantes : question $1:$ connaissance des rôles de sexe (par exemple, avec «routier», « ce sont surtout les femmes ou les hommes qui font ce travail ? »; extrémité gauche de la ligne «surtout les femmes», extrémité droite «surtout les hommes »; question 2 : possibilité de transgresser les rôles de sexe (par exemple, «si une femme a envie de faire comme travail routier, est-ce qu'elle peut ? »; extrémité gauche « pas du tout possible », extrémité droite «tout à fait possible»); question 3 : évaluation morale de la transgression (par exemple, « est-ce que c'est bien ou mal pour une femme de faire routier? $»$; extrémité gauche «pas bien du tout», extrémité droite «très bien»); question 4 : évaluation affective de la transgression (par exemple, «est-ce que tu aimerais bien être l'ami d'une femme qui serait routier ? »; extrémité gauche «j'aimerais pas du tout», extrémité droite «j'aimerais beaucoup »).

- Partie 2. Explications des différences entre sexes. En face de chacune des 6 affirmations, les sujets devaient indiquer leur degré d'accord avec les trois types d'explications (biologiques, sociales, psychologiques). Pour cela, ils se positionnaient sur une ligne de $9 \mathrm{~cm}$, l'extrémité gauche correspondant à «pas du tout d'accord», l'extrémité droite à «tout à fait d'accord». Le mode de passation était identique à l'étude 1 (deux sessions, lors de chaque session, les sujets passaient successivement la moitié des parties 1 et 2 , division des sujets en un groupe A (session 1, questions portant sur les cibles de sexe féminin ; session 2, questions sur les cibles de sexe masculin) et un groupe B (session 1, 
cibles de sexe masculin; session 2, cibles de sexe féminin), puis entretien).

\section{Résultats}

Comme dans l'étude 1, il n'y a pas de différences entre les groupes A et B.

\section{Partie 1. Flexibilité des rôles de sexe}

Les analyses de variance montrent qu'il n'y a pas de différences significatives entre les trois activités «féminines » et les trois activités «masculines». Nous avons donc calculé deux scores moyens, l'un pour les activités «féminines », l'autre pour les activités «masculines ». En conséquence, les résultats se basent sur une analyse de la variance : 2 (âge des sujets) $\times 2$ (sexe des sujets) $\times 2$ (caractère sexué des activités), le dernier facteur étant une mesure répétée. Les scores varient entre 0 et 9 . On ne constate pas d'effets principal ni d'interaction du sexe des sujets.

\section{- Connaissance des rôles de sexe}

On note un effet d'interaction simple âge des sujets $\mathrm{x}$ caractère sexué des activités $(\mathrm{F}(1,116)=$ 9,19, $\mathrm{p}<.003)$ : les enfants, par rapport aux adultes, accentuent davantage le caractère masculin des activités «masculines» (enfants $=7,41$; adultes $=6,57$; post-hoc $: \mathrm{p}<.006$ ) (axe allant de 0 : «surtout les femmes» à 9 : «surtout les hommes »). Ce n'est pas le cas des activités « féminines » pour lesquelles l'évaluation des enfants $(2,86)$ n'est pas significativement différente de l'évaluation des adultes $(3,05 ;$ post-hoc $: \mathrm{p}<.17$ ns) (voir tableau 3).

\begin{tabular}{lccc}
\hline & & Enfants & Adultes \\
\hline $\begin{array}{l}\text { Connaissance } \\
(0: \text { surtout femmes ; }\end{array}$ & AF & 2,86 & 3,05 \\
\cline { 2 - 4 } $9:$ surtout hommes) & AM & 7,41 & 6,57 \\
\hline $\begin{array}{l}\text { Possibilité } \\
\text { transgression } \\
(0: \text { pas possible ; }\end{array}$ & AF & 4,70 & 4,39 \\
\cline { 2 - 4 }$:$ possible $)$ & AM & 4,10 & 3,82 \\
\hline $\begin{array}{l}\text { Évaluation morale } \\
(0: \text { pas bien ; } 9: \text { bien })\end{array}$ & AF & 5,38 & 6,06 \\
\hline $\begin{array}{l}\text { Évaluation affective } \\
(0: \text { ne pas être l'ami ; }\end{array}$ & AF & 6,50 & 5,99 \\
\cline { 2 - 4 } $9:$ être l'ami) & AM & 5,93 & 6,00 \\
\hline
\end{tabular}

Tableau 3. Flexibilité des rôles de sexe selon l'âge. Notes moyennes par question selon le caractère sexué des activités (AF : activités «féminines »; AM : activités « masculines ») (notes comprises entre 0 et 9 ).
- Possibilité de la transgression (mesure traditionnelle de la flexibilité)

On note un effet principal du caractère sexué des activités $(\mathrm{F}(1,116)=9,53, \mathrm{p}<.0025)$ : les sujets considèrent davantage possible pour un homme de réaliser une activité «féminine » $(4,55)$ que pour une femme de réaliser une activité «masculine » $(3,96)$ (axe allant de $0:$ «pas du tout possible » à 9 : «tout à fait possible »). En revanche, il n'y a pas d'effets simples ou d'interaction de l'âge des sujets.

\section{- Évaluation morale de la transgression}

On constate un effet principal du caractère sexué des activités $(\mathrm{F}(1,116)=7,84 \mathrm{p}<.005)$, les sujets jugent moins bien la réalisation d'activités « masculines » par des femmes $(5,25)$ que la réalisation d'activités «féminines » par des hommes $(5,72)$ (axe allant de 0 « pas bien du tout » à 9 « très bien »). De même, il y a un effet principal de l'âge des sujets $(F(1,116)=22,61, p<.0001)$, les adultes étant plus tolérants $(6,02)$ que les enfants $(4,94)$. Plus précisément, on constate un effet d'interaction simple âge des sujets $\mathrm{x}$ caractère sexué des activités $(\mathrm{F}(1,116)=5,21, \mathrm{p}<.024)$, les enfants jugent mieux les transgressions pour les activités «féminines » (hommes réalisant une activité «féminine ») $(5,38)$ que pour les activités « masculines » (femmes réalisant une activité «masculine») $(4,50$; post-hoc : p <.036), ce qui n'est pas le cas des adultes, qui sont aussi tolérants pour les transgressions pour les activités «féminines » $(\mathrm{m}=$ $6,06)$ que pour les activités «masculines » $(\mathrm{m}=$ $5,99$; post-hoc : $\mathrm{p}<.60 \mathrm{~ns})$.

\section{- Évaluation affective de la transgression}

Il n'y a pas d'effets significatifs, ni principaux, ni d'interactions des différentes variables.

\section{Partie 2. Explications des différences entre sexes}

Des analyses de variance montrent qu'il n'y a pas de différences significatives entre les réponses aux trois activités «féminines »d'une part, entre les réponses aux trois activités «masculines» d'autre part. Nous avons donc calculé deux scores moyens, l'un pour les activités «féminines », l'autre pour les activités « masculines ». En conséquence, les données qui suivent se basent sur une analyse de la variance : 2 (âge des sujets) $\times 2$ (sexe des sujets) $\times 2$ (caractère sexué des activités) $\times 3$ (type d'explications), les deux derniers facteurs étant des mesures répétées. Les scores varient entre 0 (pas du tout d'accord) et 9 (tout à fait d'accord). Cette analyse de la variance montre qu'il n'y a pas d'effet principal ou d'interaction du sexe des sujets.

L'analyse statistique met en évidence trois effets principaux : $1^{\circ}$ un effet de l'âge des sujets $(\mathrm{F}(1,116)=$ 
$21,81, \mathrm{p}<.0008) ; 2^{\circ}$ un effet du caractère sexué des activités $(\mathrm{F}(1,116)=4,05, \mathrm{p}<.045) ; 3^{\circ}$ un effet du type d'explications $(\mathrm{F}(2,232)=5,34, \mathrm{p}<.005)$ (voir tableau 4). On remarque que les notes d'accord sont plus élevées chez les enfants $(6,72)$ que chez les adultes $(5,53)$, pour les activités « féminines » $(6,25)$ que pour les activités «masculines» $(6,01)$, et pour les explications psychologiques $(6,62)$ que pour les explications biologiques $(6,13)$ et les explications sociales $(5,62)$. Pour ces trois types d'explications, les comparaisons post-hoc montrent que seul l'écart entre les explications psychologiques et sociales est significatif (post-hoc : $\mathrm{p}<.002$ ). On relève deux effets d'interaction simple $: 1^{\circ}$ un effet âge des sujets $\times$ caractère sexué des activités $(F(1,116)=7,81$, $\mathrm{p}<.006)$ : les enfants ont des notes plus élevées pour les activités «féminines» $(7,00)$ que pour les activités «masculines » $(6,44 ;$ post-hoc $: \mathrm{p}<.005)$, les notes n'étant pas significativement différentes chez les adultes (activités «féminines » $=5,49$; activités «masculines » $=5,58 ;$ post-hoc $: \mathrm{p}<.53, \mathrm{~ns}) ; 2^{\circ}$ un effet âge des sujets $\times$ type d'explications $(\mathrm{F}(2,232)=$ $23,62, \mathrm{p}<.0001)$ : les enfants sont plus d'accord que les adultes avec les explications biologiques (enfants $=7,00 ;$ adultes $=5,20 ;$ post-hoc $:$ $\mathrm{p}<.0002$ ) et psychologiques (enfants $=8,03$; adultes $=5,35$; post-hoc $: \mathrm{p}<.0006$ ). À l'inverse, les adultes, davantage que les enfants, sont plus d'accord avec les explications sociales (adultes $=6,06$; enfants $=5,08 ;$ post-hoc $: \mathrm{p}<.042$ ). Enfin, on note un effet d'interaction double, âge des sujets $\times$ caractère sexué des activités $\mathrm{x}$ type d'explications $(\mathrm{F}(2,232)=3,11, \mathrm{p}<.046)$ : les enfants sont moins d'accord avec les explications sociales pour les activités «masculines» $(4,47)$ que pour les activités «féminines» $(5,61$, post-hoc $: \mathrm{p}<.003)$, contrairement aux adultes, qui ne font pas de différences (explications sociales activités « masculines » $=6,15$; explications sociales activités «féminines » $=5,98$; post-hoc : $\mathrm{p}<.27 . \mathrm{ns})$.

\begin{tabular}{llll}
\hline & \multicolumn{1}{c}{$\begin{array}{c}\text { Type } \\
\text { d'explication }\end{array}$} & Enfants & Adultes \\
\hline $\begin{array}{l}\text { Activités } \\
\text { «féminines » }\end{array}$ & Biologique & 7,33 & 5,17 \\
\cline { 2 - 4 } & Sociale & 5,61 & 6,10 \\
\cline { 2 - 4 } & Psychologique & 8,07 & 5,19 \\
\hline Activités \\
«masculines 》 & Biologique & 6,81 & 5,23 \\
\cline { 2 - 4 } & Sociale & 4,47 & 6,31 \\
\cline { 2 - 4 } & Psychologiques & 8,02 & 5,20 \\
\hline
\end{tabular}

Tableau 4. Notes moyennes selon l'âge aux trois types d'explications pour les activités «féminines》 et «masculines" (notes comprises entre 0: pas du tout d'accord et 9 : tout à fait d'accord).
Par ailleurs, des analyses de corrélation (coefficients de Bravais-Pearson) font apparaître un lien entre explications biologiques et explications sociales, positif chez les enfants $(\mathrm{Rbp}=+0,39$, $\mathrm{p}<.002)$, et négatif chez les adultes $(\mathrm{Rbp}=-0,33$, $\mathrm{p}<.01)$. En revanche, tant chez les enfants que chez les adultes, il n'y a pas de corrélations entre les explications psychologiques et les deux autres types d'explications.

Liens entre flexibilité des rôles de sexe et explications des différences entre sexes

Suite à des analyses préalables, nous avons regroupé certaines données. Dans la partie 1, par la création d'un indice de flexibilité totale résultant du regroupement des mesures «traditionnelle», «morale» et «affective» de la transgression des rôles de sexe ; dans la partie 2, par le regroupement des activités «féminines» et «masculines ». Les résultats montrent que la flexibilité des rôles de sexe est corrélée (coefficients de Bravais-Pearson), chez les adultes, négativement pour les explications biologiques, positivement pour les explications psychologiques. En revanche, ces corrélations ne sont pas observées chez les enfants. D'un autre côté, on ne constate pas de corrélations significatives, tant chez les adultes que chez les enfants, entre la flexibilité et les explications sociales (voir tableau 5).

\section{Discussion}

Cette seconde étude, mise en œuvre pour tester différentes causes possibles de l'absence de liens entre flexibilité des rôles de sexe et type d'explications des différences entre sexes dans l'étude 1, permet d'écarter certains facteurs avancés lors de la discussion de la première étude. D'abord, il ne semble pas que l'absence de liens dans l'étude 1 soit due à une mesure trop simple puisque, avec un mode de mesure plus fin, on constate de nouveau chez les enfants cette absence. Ensuite, l'hypothèse de liens entre flexibilité et explications semble valide dans la mesure où il se manifeste, dans cette étude 2, chez les adultes. Cela suggère que chez les enfants flexibilité des rôles de sexe et explications des différences entre sexes, contrairement à ce qui se passe chez les adultes (voir à l'adolescence si l'on se réfère à la recherche de Neff et TerrySchmitt, 2002), ne sont pas coordonnées. On remarque, par ailleurs, des résultats similaires, dans l'étude 1 et dans l'étude 2, chez les enfants. Ainsi, en ce qui concerne la flexibilité des rôles de sexe, les enfants sont également, dans l'étude 2, généralement plus tolérants pour les transgressions réalisées par des adultes hommes que par des adultes femmes. De même, au niveau des explications des différences entre sexes, d'une part, les enfants privilégient de nouveau les explications psychologiques, d'autre part, explications biologiques et 


\begin{tabular}{lcccccc}
\hline & \multicolumn{3}{c}{ Explications biologiques } & Explications sociales & \multicolumn{2}{c}{$\begin{array}{c}\text { Explications } \\
\text { psychologiques }\end{array}$} \\
\cline { 2 - 7 } & Enfants & Adultes & Enfants & Adultes & Enfants & Adultes \\
\hline Flexibilité des rôles de sexe & $+0,05$ & $-0,39^{*}$ & $+0,04$ & $+0,14$ & $+0,17$ & $+0,41^{*}$ \\
\hline
\end{tabular}

Tableau 5. Corrélations entre la flexibilité des rôles de sexe et les types d'explications selon l'âge $\left(^{*}\right.$ : significatif à $\mathrm{p}<.05)$

sociales sont aussi liées positivement. Enfin, en ce qui concerne précisément l'étude 2 , on remarque que les adultes sont plus «flexibles» que les enfants et adhèrent davantage aux explications sociales.

\section{DISCUSSION GÉNÉRALE}

Cette recherche, constituée de deux études, qui avait pour but d'aborder la question de l'évolution et des liens entre flexibilité des rôles de sexe et explications des différences entre sexes, met en évidence un certain nombre de points. Pour la flexibilité des rôles de sexe (partie 1), nous avions fait l'hypothèse (hypothèse 1), en nous fondant sur l'étude de Levy et coll. (1995), qu'avec l'âge, la flexibilité dans ses aspects traditionnels (possibilité de transgression et relativité culturelle) augmenterait, tandis que la flexibilité dans ses aspects « évaluatifs » (jugements moral et affectif) resterait constante. Cette hypothèse n'est que partiellement validée : les deux types de flexibilité augmentent ; toutefois, la flexibilité, dans ses aspects traditionnels, augmente généralement plus que la flexibilité dans ses aspects évaluatifs. Il est délicat d'expliquer ces résultats, différents de ceux de Levy et coll. (1995), notamment parce qu'un écart de dix ans sépare nos deux recherches et qu'elles portent sur deux populations différentes d'enfants (américaine dans leur recherche, française dans la nôtre). Par ailleurs, on constate que les enfants sont généralement plus flexibles à l'égard des hommes exerçant des métiers qualifiés de féminins qu'à l'égard des femmes exerçant des métiers qualifiés de masculins. Cela rejoint les travaux de psychologie sociale (Eagly, Dickman, 1997). Pour les loisirs enfantins, on note le phénomène inverse, les enfants tendant à mieux accepter les transgressions effectuées par les filles que par les garçons. Dans ce cas, c'est peut-être la conséquence de l'intégration, par les enfants, des prescriptions sociales qui sont souvent plus rigides, en matière de rôles de sexe, pour les garçons que pour les filles (Ruble, Martin, 1998).

Pour l'explication des différences entre sexes (partie 2), nous avions fait l'hypothèse (hypothèse 2) qu'avec l'âge, les explications biologiques et psychologiques diminueraient, tandis que les explications sociales augmenteraient. C'est effectivement le cas quand on compare les enfants et les adultes. Néanmoins, si l'on se réfère uniquement aux évolutions des enfants (étude 1), on remarque que les explications psychologiques sont privilégiées et qu'elles ont tendance à augmenter jusqu'à 9 ans, pour diminuer, ensuite, chez les enfants de 11 ans. De plus, toujours chez les enfants, on note que les explications sociales et les explications biologiques se situent à un niveau relativement bas. Ces résultats, la présence importante des explications psychologiques, la présence assez faible des explications biologiques, concordent mieux avec les travaux de Carey $(1985,1995)$ qu'avec ceux de Gelman et Taylor (2000) sur l'essentialisme biologique enfantin. À ce niveau, l'étude des corrélations mérite d'être mentionnée. Si l'on se réfère à l'essentialisme biologique enfantin, influences sociales et influences biologiques sont deux logiques différentes, opposées, la première se développant au fur et à mesure que la seconde diminue. On aurait donc dû s'attendre à un lien négatif entre explications biologiques et sociales. Or, on remarque chez les 5-7 ans (la tendance s'inversant chez les plus âgés), que les explications biologiques et les explications sociales sont liées positivement. On peut dire que ces deux types d'explications ont en commun de renvoyer à des processus, internes pour les unes, externes pour les autres, qui sont au-delà du contrôle personnel de l'individu. Si elles sont liées chez les plus jeunes, c'est, peut-être, parce qu'à cet âge elles renvoient à l'adhésion aux stéréotypes traditionnels où la division sociale du genre (comportements considérés comme masculins ou féminins) va de pair avec la division biologique des sexes (sujets de sexe masculin ou féminin). À titre indicatif, en discutant avec les enfants lors des entretiens, nous avons remarqué que les explications biologiques et sociales semblaient associées à une attitude de respect des attentes du milieu social. Quant aux explications sociales, on peut supposer qu'elles changent de signification avec l'âge. Chez les adultes de l'étude 2, d'une part, les explications biologiques et les explications sociales sont corrélées négativement, d'autre part, dans les discussions avec les sujets après l'expérience, les explications sociales étaient souvent énoncées pour 
souligner les contraintes sociales, les déterminismes sociaux. Enfin, et cela va dans le sens d'une sensibilité plus grande aux contraintes sociales avec l'âge, les explications psychologiques, qui renvoient à l'idée de libre choix, diminuent chez les adultes.

Si l'on s'intéresse, pour terminer, aux liens entre flexibilité des rôles de sexe et type d'explications des différences entre sexes, on remarque que les effets sont moins linéaires que ceux que l'on attendait (hypothèse 3 ). Dans l'étude 1 , on constate un seul lien, uniquement chez les enfants de 11 ans, en l'occurrence, un lien positif entre flexibilité et explications sociales. Cela semble signifier que chez les enfants, du moins jusqu'à 11 ans, la flexibilité ne s'inscrit pas encore dans une lecture explicative de l'origine des différences entre sexes. Chez les adultes (étude 2), il semble que ce soit le cas, car on observe bien un certain nombre de liens entre flexibilité et type d'explications. Toutefois, ces liens sont en partie différents de ceux que l'on avait envisagés. Si, comme nous le supposions, des liens négatifs existent entre flexibilité des rôles de sexe et explications biologiques, les liens positifs se situent, contrairement à notre attente, non pas entre explications sociales et flexibilité des rôles de sexe, mais entre cette dernière et les explications psychologiques. Ainsi, chez les adultes, l'explication biologique, en renvoyant à quelque chose qui existe a priori et nous dépasse, semble contribuer à justifier une vision relativement figée des rôles de sexe et, à l'inverse, l'explication psychologique, peutêtre parce qu'elle met l'accent sur notre propre vouloir, nos propres choix, s'associe à l'idée que les rôles de sexe peuvent être modulables et plus ou moins respectés par les individus. En définitive, si l'on compare les résultats des enfants et ceux des adultes, les liens semblent ne pas renvoyer à la même chose. Chez les enfants les plus âgés dans notre recherche (enfants de 11 ans de l'étude 1), la flexibilité s'associe à une prise en compte des influences sociales (corrélation positive entre flexibilité et explications sociales), tandis que, chez les adultes, cette flexibilité correspond à une représentation psychologique, et non biologique, de l'origine des différences entre sexes (corrélation positive entre flexibilité et explications psychologiques).

Cette recherche avait but de comprendre comment les enfants et les adultes appréhendent les rôles de sexe, qui contribuent, pour une part, aux inégalités entre les sexes. Les résultats montrent que les jeunes enfants adhèrent peu aux explications sociales. Cela signifie qu'ils ont peu conscience de l'influence que les facteurs sociaux ont sur la construction des comportements sexués. Par la suite, pour mieux comprendre ce qui soustend les inversions de corrélations constatées dans ces deux études selon l'âge, entre les différentes explications et entre celles-ci et la flexibilité, il conviendra d'étudier plus précisément la signification attachée, en fonction de l'âge, à ces explications et d'élargir les recherches à des populations adolescentes. Enfin, ainsi que l'indiquent Brown et Bigler (2005), il serait utile, dans une perspective appliquée, d'étudier dans quelle mesure cette flexibilité des rôles de sexe et ces explications ont une influence sur la façon dont les enfants perçoivent et jugent les discriminations entre les sexes.

\section{RÉFÉRENCES}

ARCHER (Cynthia J.).- Children's attitudes toward sexrole division in adult occupational roles, Sex Roles, $X$, 1-2, 1984, p. 1-10.

Astuti (Rita), Solomon (Gregg E.), CAREy (Susan).Constraints on conceptual development, Monographs of the Society for Research in Child Development, LXIX, 3, 2004, p. 1-135.

BEM (Sandra L.).- Genital knowledge and gender constancy in preschool children, Child Development, LX, 1989, p. 649-662.

Bourdieu (Pierre).- La domination masculine, Paris, Seuil, 1998.

Brown (Christia-S.), BIGLER (Rebecca-S.).- Children's perceptions of discrimination : a developmental model, Child Development, LXXVI, 2005, p. 533-553.
CAREY (Susan).- Conceptual change in childhood, Cambridge, MIT Press, 1985.

CAREY (Susan).- On the origin of causal understanding, dans Sperber (D.), Premack (D.), Causal cognition: A multidisciplinary debate, Oxford, Clarendon Press, 1995, p. 268-302.

CARter (D. Bruce), Patterson (Charlotte J.).- Sex roles as social conventions : the development of children's conceptions of sex-role stereotypes, Developmental Psychology, XVIII, 1982, p. 812-824.

Chiland (Colette).- Nouveaux propos sur la construction de l'identité sexuée, Journal de la psychanalyse de l'enfant, XXXIII, 2003, p. 105-122.

DAfFlon-Novelle (Anne).- Pourquoi les garçons ne jouent pas aux Barbies et les filles ne se déguisent pas en cow-boys? Liens entre constance de genre et 
perception des stéréotypes de genre chez des enfants âgés de 3 à 7 ans, dans Actes du $5^{\text {e }}$ Congrès international de psychologie sociale de langue française, Lausanne, 1-4 septembre 2004, p. 417-418.

Deaux (Kay), Lafrance (Marianne).- Gender, dans Fiske (S. T.), Gilbert (D. T.), The Handbook of Social Psychology, Boston, McGraw-Hill, 1998, p. 788-827.

EAGLY (Alice H.), DickMAN (Amanda B.).- The accuracy of gender stereotypes : a dilemma for feminism, Revue internationale de psychologie sociale, X, 2, 1997, p. 11-30.

EMMERICH (Walter).- Nonmonotonic developmental trends in social cognition : the case of gender identity, dans Straub (S.), U-Shaped behavioural growth, NewYork, Academic Press, 1982, p. 249-269.

Gelman (Susan A.).- The essential child: origins of essentialism in everyday thought, Oxford, Oxford University Press, 2003.

Gelman (Susan A.), TAYlor (Marianne G.).- Gender essentialism in cognitive development, dans Miller (P. H.), Toward a feminist developmental psychology, New York, Routledge, 2000, p. 169-190.

Helwig (Charles C.).- It is ever ok to exclude on the basis of race or gender? The role of context, stereotypes, and historical change, Monographs of the Society for Research in Child Development, Serial no. 271, LXVII, 4, 2002, p. 120-129.

Hort (Barbara E), FAgOt (Beverly I.), LeINBACH (Mary D.).- Are people's notions of maleness more stereotypically framed than their notions of femaleness, Sex Roles, XXIII, 3-4, 1990, p. 197-212.

INSEE-DROITS DES FEMMES.- Les hommes et les femmes : regards sur la parité, Paris, Insee, 2001.

Johnson (Susan C.), Solomon (Gregg E.).- Why dogs have puppies and cats have kittens : the role of birth in young children's understanding of biological origins, Child Development, LXVIII, 1997, p. 404-419.

KoHLBERG (Lawrence).- A cognitive developmental analysis of children's sex-role concepts and attitudes, dans Maccoby (E. E.), Jacklin (C. N.), The psychology of sex differences, Stanford, Stanford University Press, 1966, p. 82-173.

LE MANER-IDrIssi (Gaïd).- L'identité sexuée, Paris, Dunod, 1997.

LeVy (Gary D.), TAYloR (Marianne G.), Gelman (Susan A.).- Traditional and evaluative aspects of flexibility in gender roles, social conventions, moral rules, and physicial laws, Child Development, LXVI, 2, 1995, p. 515-531.

LibEN (Lynn S.), Bigler (Rebecca S.).- The developmental course of gender differentiation, Monographs of the Society for Research in Child Development, LXVII, 2, 2002.

Lloyd (Barbara), Stroyan (James).- Preschool children's genital knowledge : does Bem's report of a male deficit survive replication ? British Journal of Developmental Psychology, XII, 1994, p. 251-258.

LORENZI-CIOLDI (Fabio).- Les représentations des groupes dominants et dominés : collections et agrégats, Grenoble, Presses universitaires de Grenoble, 2002.

MARTin (Carol L.).- Attitudes and expectations about children with non-traditional and traditional gender roles, Sex Roles, XXII, 1990, p. 151-165.

Martin (Carol L.), PARKer (Sandra).- Folk theories about gender and race differences, Personality and Social Psychology Bulletin, XXI, 1995, p. 45-57.

Martin (Carol L.), Ruble (Diane N.), Szkrybalo (Joel).- Cognitive theory of early gender development, Psychological Bulletin, CXXVIII, 6, 2002, p. 903-933.

Miller (Linda), BudD (Jacqueline).- The development of occupational sex-role stereotypes, occupational preferences and academic subject preferences in children at ages 8, 12 and 16, Educational Psychology, XIX, 1, 1999, p. 17-35.

NefF (Kristin D.), TERRY-SchMITT (Lisa N.).- Youths' attributions for power-related gender differences : nature, nurture, or God? Cognitive Development, XVII, 2002, p. 1185-1202.

Piaget (Jean).- La représentation du monde chez l'enfant, Paris, Presses Universitaires de France, 1926.

REID (Guynel M.).- Children's occupational sex-role stereotyping in 1994, Psychological Reports, LXXVI, 1995, p. 1155-1165.

Ruble (Diane N.), Martin (Carol L.).- Gender development, dans Damon (W.), Eisenberg (N.), Handbook of Child Psychology, III, New-York, John Wiley and Sons, 1998, p. 933-1016.

Smetana (Judith G).- Preschool children's conceptions of sex-role transgressions, Child Development, LVII, 4, 1986, p. 862-871.

SMith (Jacqui), Russell (Graeme).- Why do males and females differ? Children's beliefs about sex differences, Sex Roles, XI, 1984, p. 1111-1120.

StOdDart (Trish), TurIEL (Elliot).- Children's concepts of cross-gender activities, Child Development, LVI, 1985, p. 1241-1552.

Szkrybalo (Joel), Ruble (Diane-N.).- « God made me a girl » sex-category constancy judgments and explanation revisited, Developmental Psychology, $X X X V, 2$, 1999, p. 392-402.

TAP (Pierre).- Masculin/féminin chez l'enfant, Toulouse, Privat, 1985.

TAYLOR (Marianne G.).- The development of children's belief about social and biological aspects of gender, Child Development, LXVII, 4, 1996, p. $1555-1571$.

ULLIAN (Dorothy Z.).- The development of conception of masculinity and feminity, dans Lloyd (B.), Archer (C. J.), Exploring sex differences, Londres, Academic Press, 1976, p. 25-47. 